\title{
Active Vibration Control of Cantilever Beam with Macro-Fibre Composite Actuator Using Time- Delayed Genetic Algorithm Controller
}

\author{
Xue Xiaomin \\ Department of Civil Engineering, Xi'an Jiaotong University, Xi'an, China, 710049. \\ Wu Xiaohong \\ School of Aerospace, Xi'an Jiaotong University, Xi'an, China, 710049.
}

\author{
Sun Qing \\ Department of Civil Engineering, Xi'an Jiaotong University, Xi'an, China, 710049.
}

\begin{abstract}
(Received 8 November 2014; accepted 30 September 2015)
As a type of intelligent active devices, macro-fibre composite (MFC) actuator serves as an ideal candidate for diverse applications in structural vibration suppression owing to its high performance, durability, and flexibility in a cost-competitive device. However, its development is usually plagued by MFC's complicated hysteretic property that creates difficulties for deducing the relationship between applied voltage and control force. Additionally, its application is also conditioned by time delay, which not only degrades the performance but also induces instability of the control system. In view of this, an active control method is proposed here for mitigating distributed parameter systems actuated by MFC patch. First, an electric-mechanical characteristic experiment of the MFC is carried out to obtain its mathematic relation between applied voltage and relative strain, which can lay a basic foundation for the control algorithm design of MFC systems. Next, based on uncoupled motion equation of a distributed system, an optimized time-delayed control law is proposed by using a genetic algorithm (GA) procedure. Finally, numerical examples and corresponding experimental cases are utilized to verify the proposed approaches capable of providing satisfactory control effect with different time delays. The present study can cast light on the development of other novel smart devices and their controlled systems.
\end{abstract}

\section{INTRODUCTION}

Nowadays, engineering structures such as aerospace structures, architectural structures, and vehicle engineering structures are becoming more and more large and complex as a result of rapid development of science and technology. ${ }^{1}$ Their mitigation of the damage induced by severe dynamic loads becomes a research subject of paramount importance. Recently, many researchers have directed to develop various smart materials for controlling vibration systems due to their reliability and adaptability. ${ }^{2,3}$ Smart materials such as piezoelectric materials, especially PZT ceramics, are used in many applications owing to their high resolution, high bandwidth, low cost, and the simple treatment that piezoceramics offer. ${ }^{4}$ However, the brittle nature of ceramics makes them vulnerable in vibration structures with large amplitudes, and also affects their ability to conform to curved surfaces. ${ }^{5}$ Recently, piezocomposite transducers have been gaining more and more attention due to their flexibility of bearing greater deformation. It is because the piezocomposite is composed of piezoelectric fibres mixed with a softer passive epoxy matrix. ${ }^{6,7}$ The macro-fibre composite (MFC) constructed and developed by NASA Langley Center is the most widely used piezocomposite transducer since $1996 .^{8}$ It is the leading low-profile actuator offering high performance, durability, and flexibility in a cost-competitive device when compared with piezoceramic actuators. ${ }^{7}$ The MFC actuator provides these merits and shows great potential in structural vibration control, structural health monitoring systems and other fields. However, MFC's development is still plagued by its complicated hysteretic property, which creates difficulties for deducing the relationship between applied voltage and control force.

In addition to the selected device in control systems, another problem is paying attention to time delays. As is well known, time delay inevitably exists in control systems that should not be ignored blindly. In control systems, if time delay is not properly handled, it may have worse or even adverse effects on the control actions. For instance, it may cause degradation of control efficiency or even instability of control systems. ${ }^{9}$ In order to decrease or eliminate the negative effect of time delay in controlled systems, time-delayed compensation method is primitively proposed to deal with small delays. ${ }^{10,11}$ Abdel has used the method to ensure galloping suppression of controlled suspension bridge. ${ }^{12}$ Shao applied the method to suppress the first two mode vibrations of a cantilever-like structure. ${ }^{13}$ According to large time-delay systems, some new methods have emerged in large numbers that employ $H_{\infty}$ control. ${ }^{14,15}$ fuzzy control combined neural network, ${ }^{16}$ modified optimal control method ${ }^{17}$ etc., consequently. These time-delayed methods mainly remain in the phase of theoretical research, which are separately verified valid by large number of simulation examples. In this sense, more experiments should be carried out to demonstrate their effectiveness further.

Based on the preceding research background, this paper proposes a time-delayed controller using genetic algorithm (GA) to efficiently restrict a distributed beam system with MFC actu- 


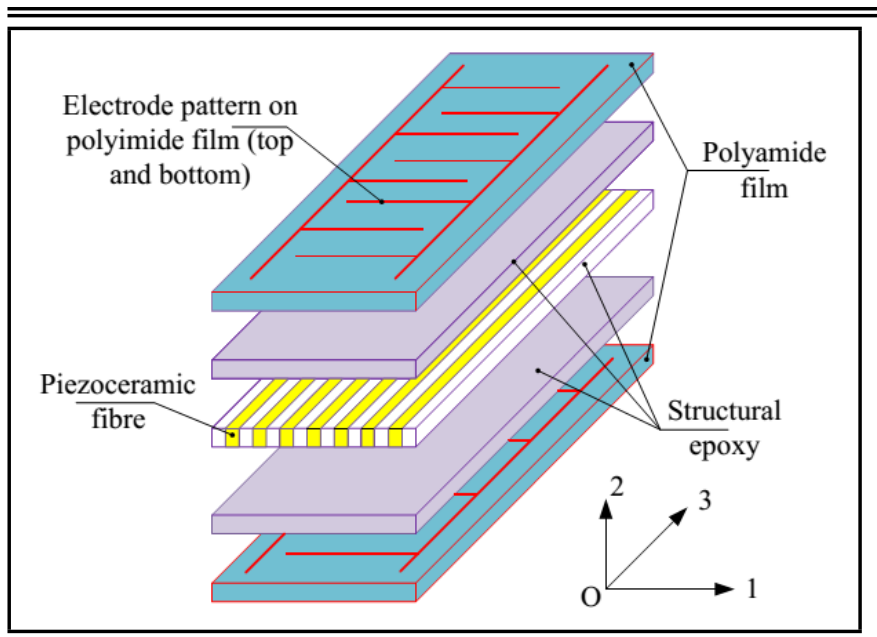

Figure 1. MFC actuator construction. ${ }^{8}$

ator. The feasibility and efficiency of the intelligent controller are both verified through experiment and numeric calculation.

The paper is organized as follows. Section 2 introduces electric-mechanical modelling of the MFC based on experiment study. Section 3 represents an active GA controller to mitigate cantilever beam that is actuated and excited by the MFC patch. Section 4 gives introduction of the vibration experiment for controlling the cantilever beam made of leadaluminium alloy. In Section 5, numerical and experimental studies are carried out to verify the validity of the proposed approaches. Final conclusion is drawn in Section 6.

\section{ELECTRIC-MECHANICAL BEHAVIOUR OF THE MFC ACTUATOR}

The MFC transducers are laminated, planar actuation devices that employ rectangular cross-section, unidirectional piezoceramic fibres embedded in a thermosetting polymer matrix. The transducers made of MFC generally consist of polyamide films with interdigited electrodes that are glued on the top and bottom of piezoceramic fibres. Its configuration can be found in Fig. 1.8 The electrodes deliver the electric field required to activate the piezoelectric effect in the fibres and allow invoking the stronger longitudinal piezoelectric effect along the length of the fibres.

\subsection{Constitutive Equations}

The objective in this article is to effectively control continuous systems subjected by MFC actuators. Hence it is very pivotal to characterize the electromechanical behaviour of the actuator where material nonlinearities should be well considered. This task can be visualized as determining, either experimentally or analytically, the material properties required by the actuator form of the linear constitutive relationship that governs the behaviour of the MFC, ${ }^{18}$

$$
\varepsilon_{i}=s_{i j}^{E} \sigma_{j}+d_{k i} E_{k}, \quad(i, j=1,2,3,4,5,6 ; k=1,2,3) ;
$$

where $\varepsilon_{i}$ represents the total strain, $\sigma_{j}$ represents the mechanical stress, $E_{k}$ is the applied electric field, $s_{i j}^{E}$ is the mechanical compliance tensor under short circuit electrical boundary conditions, and $d_{k i}$ is the piezoelectric strain tensor. It is noted that subscript $i$ and $j$ indicate the direction of strain and stress components, and $k$ is the direction of electric field.
As shown in Fig. 1, the MFC is poled in the 3-direction that is parallel to the PZT fibres, while the electrode fingers are aligned in the 1-direction where Eq. (1) can be also expressed as

$$
\begin{aligned}
\left\{\begin{array}{l}
\varepsilon_{1} \\
\varepsilon_{2} \\
\varepsilon_{3} \\
\varepsilon_{4} \\
\varepsilon_{5} \\
\varepsilon_{6}
\end{array}\right\}= & {\left[\begin{array}{cccccc}
\frac{1}{E_{1}} & -\frac{\nu_{12}}{E_{1}} & -\frac{\nu_{13}}{E_{1}} & 0 & 0 & 0 \\
-\frac{\nu_{12}}{E_{1}} & \frac{1}{E_{1}} & -\frac{\nu_{13}}{E_{1}} & 0 & 0 & 0 \\
-\frac{\nu_{13}}{E_{1}} & -\frac{\nu_{13}}{E_{1}} & \frac{1}{E_{3}} & 0 & 0 & 0 \\
0 & 0 & 0 & \frac{1}{G_{13}} & 0 & 0 \\
0 & 0 & 0 & 0 & \frac{1}{G_{13}} & 0 \\
0 & 0 & 0 & 0 & 0 & \frac{1}{G_{12}}
\end{array}\right]\left\{\begin{array}{l}
\sigma_{1} \\
\sigma_{2} \\
\sigma_{3} \\
\sigma_{4} \\
\sigma_{5} \\
\sigma_{6}
\end{array}\right\}+} \\
& {\left[\begin{array}{ccc}
0 & 0 & d_{31} \\
0 & 0 & d_{31} \\
0 & 0 & d_{33} \\
0 & d_{15} & 0 \\
d_{15} & 0 & 0 \\
0 & 0 & 0
\end{array}\right]\left\{\begin{array}{l}
E_{1} \\
E_{2} \\
E_{3}
\end{array}\right\} }
\end{aligned}
$$

where $E$ and $\nu$ are elastic modulus and Poisson's ratio, respectively, and $G$ is shear modulus that was deduced from $G=\frac{E}{2(1+\nu)}$. The use of interdigitated electrodes renders $E_{1}$ and $E_{2}$ to zero, and the only nonzero electric field is $E_{3}$. Since the MFC is a thin, planar actuator in a state of plane stress, only $\sigma_{1}, \sigma_{3}$, and $\sigma_{6}$ are nonzero, corresponding to stresses in the PZT fibre direction, transverse to the PZT fibres (in-plane), and in-plane shear stress, respectively. In this case, the constitutive relationship can be further reduced for the specific case of the MFC under general loading. With these simplifications in mind, the full form of Eq. (2) can be rewritten as

$$
\begin{aligned}
& \varepsilon_{1}=s_{11} \sigma_{1}+s_{13} \sigma_{3}+d_{31} E_{3} ; \\
& \varepsilon_{2}=s_{12} \sigma_{1}+s_{13} \sigma_{3}+d_{31} E_{3} ; \\
& \varepsilon_{3}=s_{13} \sigma_{1}+s_{33} \sigma_{3}+d_{33} E_{3} ; \\
& \varepsilon_{6}=s_{66} \sigma_{6} ;
\end{aligned}
$$

where compliance terms $s_{i j}$ are derived from $E, \nu$, and $G$. Note that the Eq. (4) represents the out-of-plane strain for the coordinate system, which is of little interest for the planar actuation of the MFC. Consequently, the out-of-plane and induced in-plane shear strains can be regarded as negligible. The remaining constitutive equations that will guide the experimental characterization approach are

$$
\begin{aligned}
& \varepsilon_{1}=s_{11} \sigma_{1}+s_{13} \sigma_{3}+d_{31} E_{3} ; \\
& \varepsilon_{3}=s_{13} \sigma_{1}+s_{33} \sigma_{3}+d_{33} E_{3} .
\end{aligned}
$$

Here, the parameters $\left(s_{11}, s_{13}, s_{33}, d_{31}\right.$, and $\left.d_{33}\right)$ that express material properties of the MFC should be determined. We have known that $s_{i j}$ can be acquired from $E, \nu$, and $G$, but $d_{31}$ and $d_{33}$ vary with physical quantities such as stress, strain, voltage, or temperature. As a result, guaranteeing the influencing factors $d_{31}$ and $d_{33}$ is a pivotal task that needs to be figured out.

\subsection{Experiment of Electric-Mechanical Behaviour}

In this paper, the selected MFC product developed by NASA Langley Center is applied to control a cantilever beam made of lead-aluminium alloy. The material properties of the MFC are revealed in Table 1. In order to investigate its electricmechanical behaviour, an experimental test for MFC patch is 


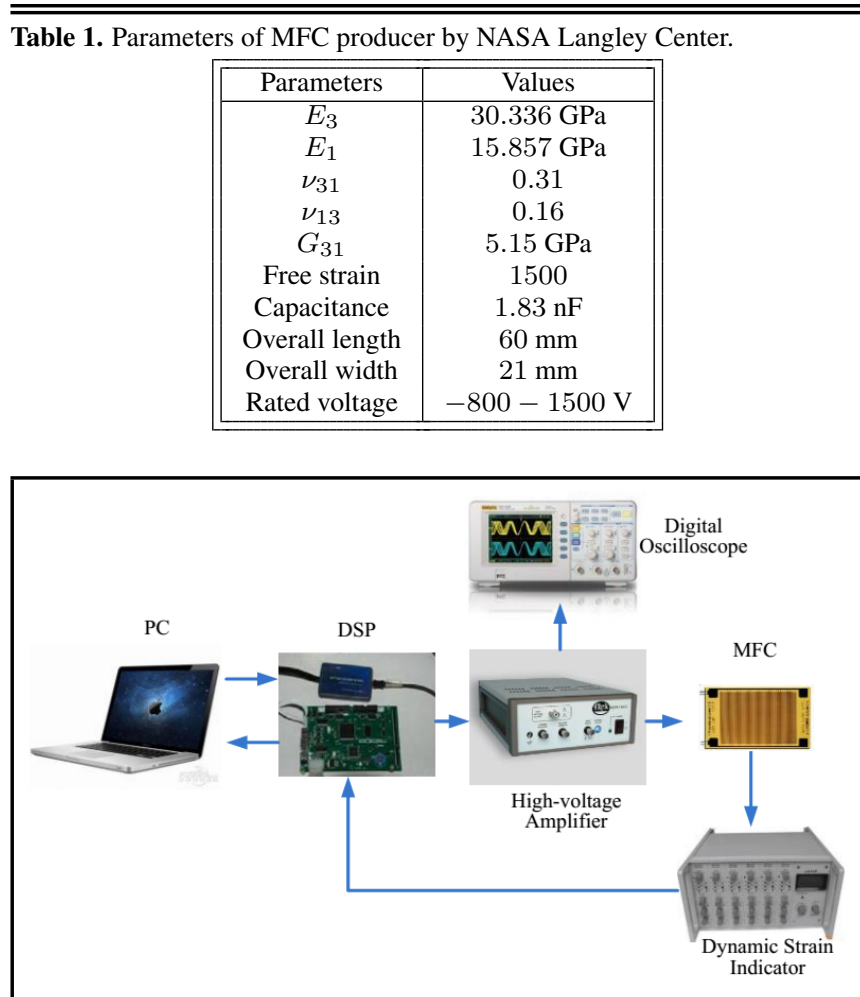

Figure 2. Schematic of the experimental setup.

implemented in State Key laboratory for Strength and Vibration of Mechanical Structures in Xi' an Jiaotong University. As depicted in Fig. 2, its operational process using various experimental apparatus and equipment is briefly explained below.

Digital signal processing (DSP) is the key component in the test. It can realize various digital signal process algorithms by special hardware multiplier, which can be viewed as the core controller in active control systems. Based on the flow chart, DSP can be used to receive the personal computer (PC) signal and transmit the received signal to a high-voltage amplifier. At the same time, DSP receives a series of strain data recorded by dynamic strain indicators and then delivers it to the PC. The MFC patch is connected with the high-voltage amplifier whose leads are connected to a digital real-time oscilloscope that demonstrates the primitive and amplifying voltage. Synchronously, strain gauges bonded in the MFC patch are also connected to dynamic strain indicators that relay strain data to the DSP.

As is known, non-uniform field distribution inevitably exists because the interdigitated electrode pattern of the MFC complicates the electric field shape. Some researchers have dedicated to calculate such a complex field distribution. In this paper, we dedicate to investigate the electric-mechanical characteristics of the MFC and propose an efficient method for modelling it. Finally these proposed approaches are applied to active vibration control systems with MFC actuators. In view of this, the complex field is simplified and averaged. Moreover, in the light of a previous study, ${ }^{18}$ the average electric field $E$ is adopted herein that is developed in the MFC actuator under the application of voltage $U$. It is defined as

$$
E=\frac{U}{d_{m}}
$$

where $d_{m}$ is the centre-to-centre interdigitated electrode spacing valued $500 \mu \mathrm{m}$. (a) Time history.

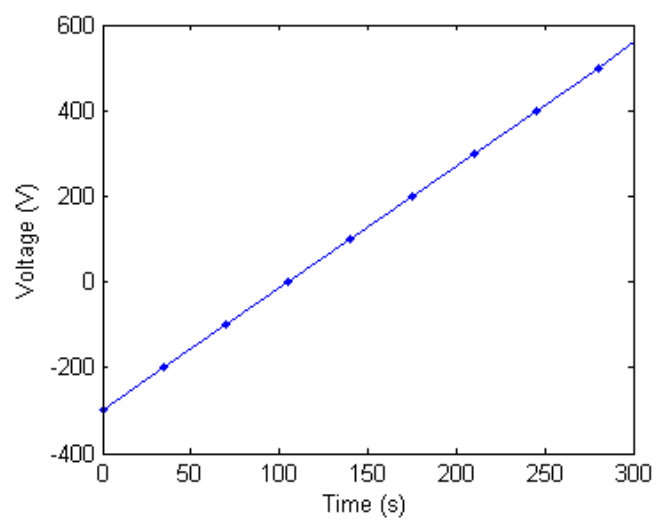

(b) Electric intensity vs. strain.

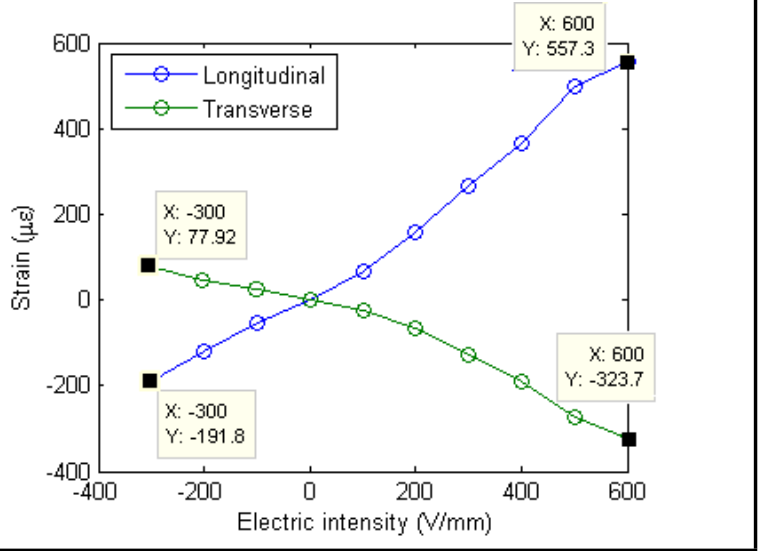

Figure 3. Electric-mechanical behaviour under constant voltages.

In this paper, an electric-mechanical characteristic experiment of MFC actuators has been carried out for two cases, one of which is under static electric field, and the other is under dynamic electric field.

\section{1) Static characteristic test}

First, we focus on the response to a monotonic increase in actuation voltage. As shown in Fig. 3(a), the MFC actuator is excited by a series of constant voltages increasing from $-300 \mathrm{~V}$ to $600 \mathrm{~V}$ at intervals of $100 \mathrm{~V}$. Figure 3(b) displays the measured longitudinal and transverse strains simultaneously. In Fig. 3(b), the measured strain has approximately linear relation with longitudinal/transverse strains where there are no hysteresis or nonlinearities present. When the electric intensity is gradually increasing, the longitudinal strain will increase within the range of $749.1 \mu \varepsilon$; however, the transverse strain will decrease with a relative small range of $401.62 \mu \varepsilon$. In this sense, the longitudinal electric-mechanical behaviour ( $d_{33}$ effect) of MFC is much outstanding than the transverse effect $\left(d_{31}\right)$, as a result it has applications such as active vibration control and structural health monitoring or shape control.

\section{2) Dynamic characteristic test}

Under the dynamic excitations, we observe the hysteretic response of the MFC. In this case, the MFC actuator is excited by a series of harmonic voltages with different frequencies and peak-to-peak voltages (VPPs). Note that the frequencies are severally designed as $0.1 \mathrm{~Hz}, 0.2 \mathrm{~Hz}, 0.3 \mathrm{~Hz}, 0.4 \mathrm{~Hz}$, $0.5 \mathrm{~Hz}, 1 \mathrm{~Hz}, 2 \mathrm{~Hz}, 5 \mathrm{~Hz}$, and $10 \mathrm{~Hz}$, and the VPPs are designed as $200 \mathrm{~V}, 400 \mathrm{~V}, 600 \mathrm{~V}, 800 \mathrm{~V}, 1000 \mathrm{~V}, 1200 \mathrm{~V}, 1400 \mathrm{~V}$, and 1600 V. As shown in Fig. 4, some representative results 


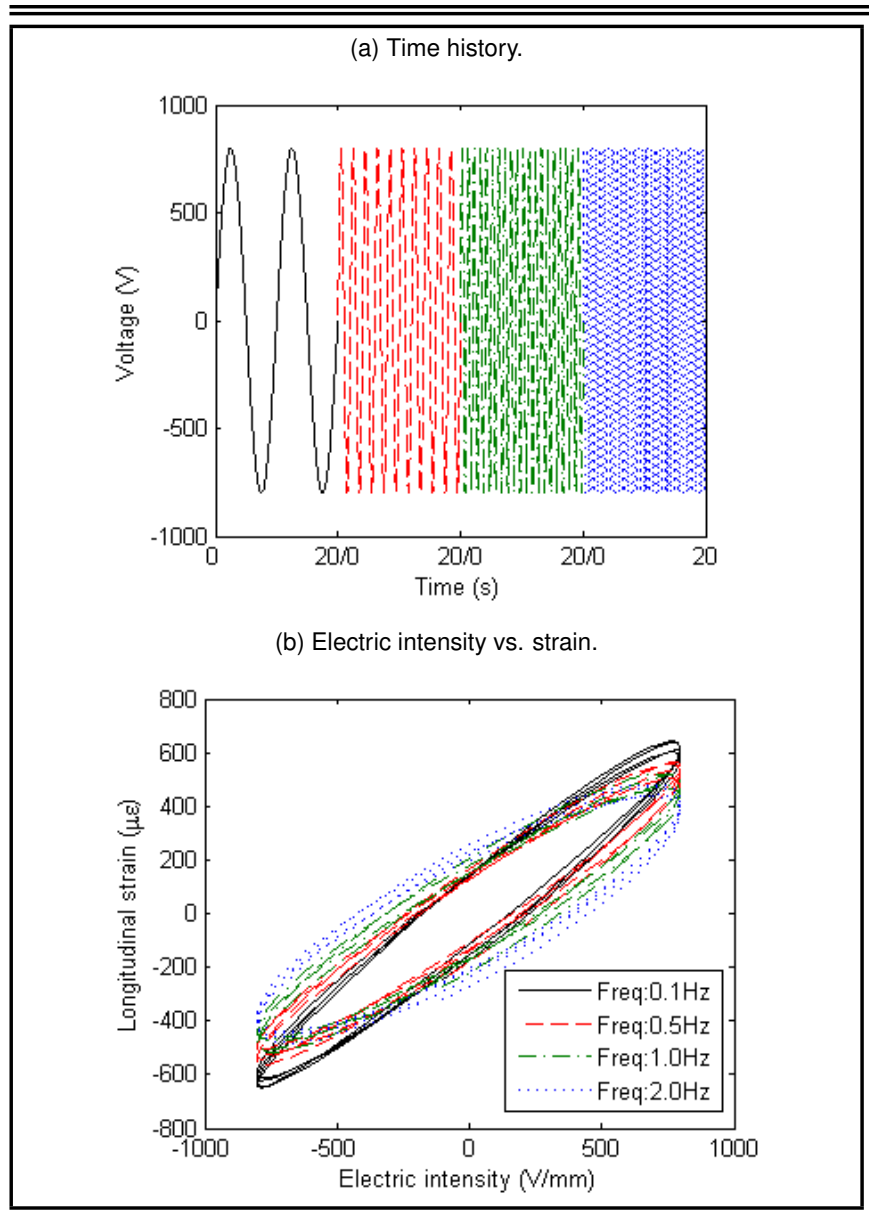

Figure 4. Electric-mechanical behaviour with different frequencies.

are derived from the cases that VPP is $1600 \mathrm{~V}$ with different frequencies of harmonic voltage input. The hysteretic curves show different hysteretic shapes with different average slopes when various voltage inputs are loaded. The principle is that voltage input with higher frequency results in fuller hysteretic loop with lower average slope. Figure 5 shows demonstration for the cases that the frequency of the applied harmonic electric field is set as $2 \mathrm{~Hz}$ under different VPPs. Note that for the longitudinal strain-electric intensity loops, the figures seem like slant ellipses maintaining a uniform shape even in different electric field. The only difference is that the ellipse becomes bigger with a higher VPP. As a result, the electric intensity-strain relationship of the MFC actuator is variegated, which must be accurately simulated by one appropriate electromechanical model.

In this test, the dynamic characteristic also reveals that the longitudinal electric-mechanical behaviour is more prominent than transverse effect. Hence, we mainly focus on the mechanical property between electric field and produced strain under the $d_{33}$ operational mode.

\subsection{Modelling of Electric-Mechanical Behaviour}

MFC has the capability to control systems owing to its electric-mechanical characteristic. After investigating its property between electric intensity and induced strain, we should establish an appropriate electric-mechanical mathematical model so as to send accurately controlling order to MFC actuator. Only if the controlling order is executed accurately and efficiently enough, the vibration of systems is controlled (a) Time history.

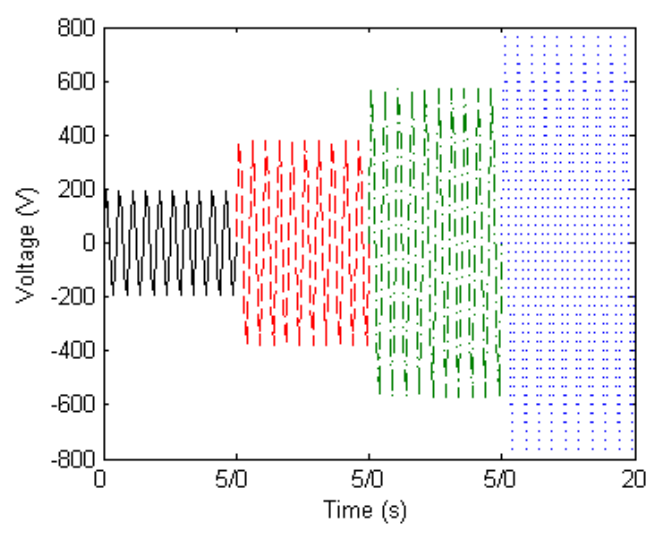

(b) Electric intensity vs. strain.

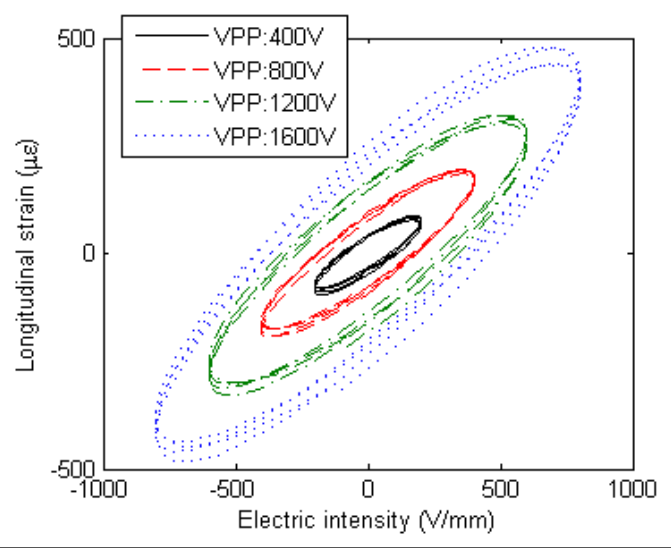

Figure 5. Electric-mechanical behaviour with different peak-to-peak voltage (VPP).

by the MFC actuators under a satisfactory state.

As observed from the experimental data, the electric mechanical behaviour represents nonlinear hysteretic characteristics. Here, the normalized version of the Bouc-Wen model introduced in previous work ${ }^{19}$ relates the output $\varepsilon(t)$ to the input $x(t)$ in the following way

$$
\varepsilon(t)=c \dot{x}(t)+k x(t)+\alpha z(t)+f ;
$$

where evolutionary variable $z$ is governed by

$$
\dot{z}(t)=A x(t)-\beta \dot{x}(t)|z(t)|^{n}-\gamma|\dot{x}(t)| z(t)|z(t)|^{n-1} ;
$$

where $\varepsilon$ is the output, which can be strain of the MFC actuator, $z$ is imaginary intermediate variable, $x$ can be the voltage strength or electric-field strength whose derivative is $\dot{x}$, and the rest alphabets including $c, k, \alpha, f, A, \beta, \gamma$, and $n$ are unknown parameters that should be identified through test data by using a optimization algorithm. The parameter $c$ is scaling connection of the proportion by the variables $\varepsilon$ and $\dot{x}$. The parameter $k$ is the scaling connection of the proportion by $\varepsilon$ and $x$. The parameter $\alpha$ is a scaling factor, and $f$ is the electric field bias applied to MFC actuator.

Based on the earlier study about performance testing and model investigation, ${ }^{20}$ the parameters can be taken as $c=0$, $f=0, \alpha=0.4579, A=1.2399$, and $\beta=1.5969$. The rest parameters $k, \gamma$, and $n$ are very sensitive to the model's output, which is dependent on the different input voltages including frequencies and supplied amplitudes. Hence, according to different VPPs from 0.2 to $0.8 \mathrm{kV}$ and frequencies from 0 to $5 \mathrm{~Hz}$, the identified parameters $k, \gamma$, and $n$ are grouped and 


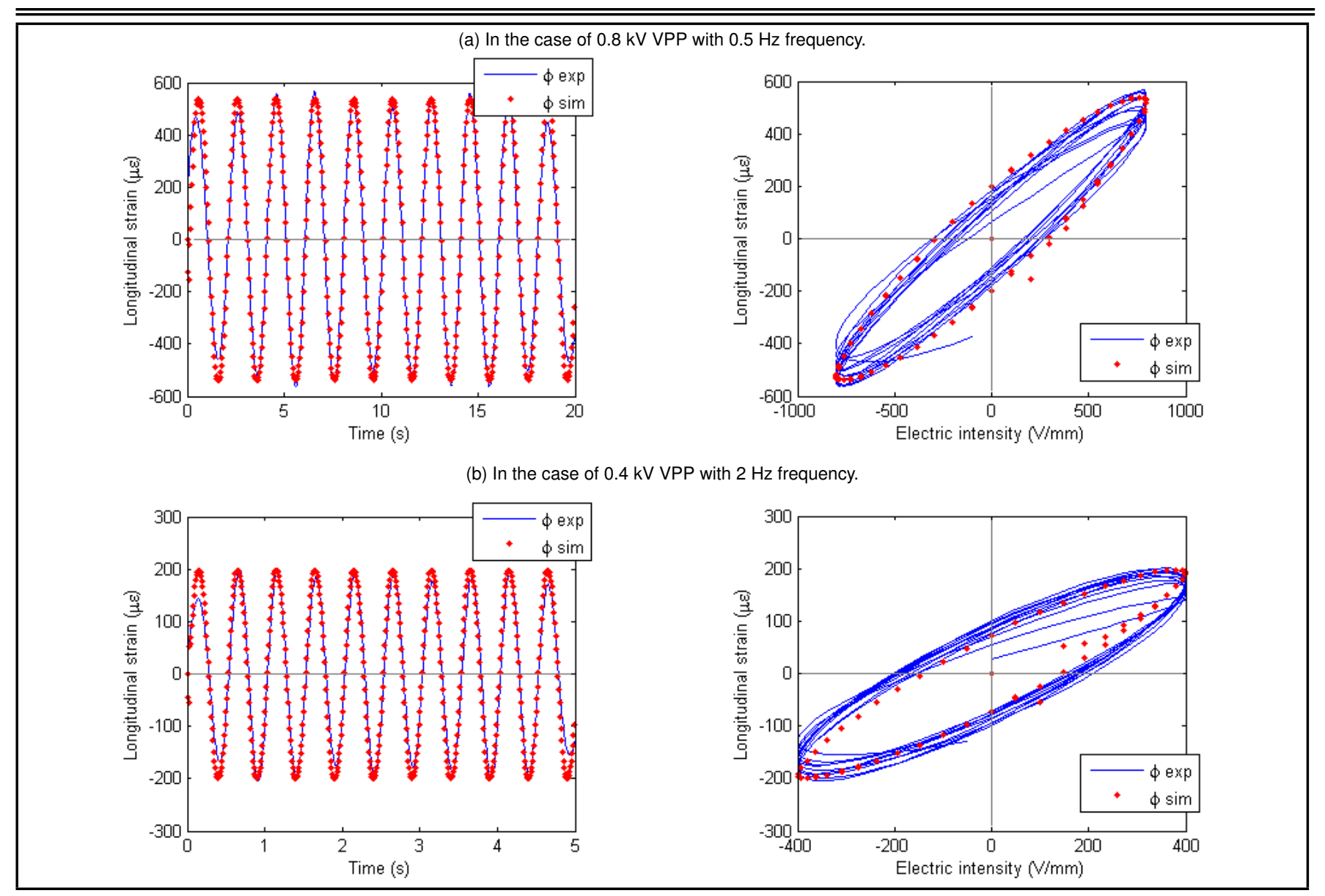

Figure 6. Comparison between experiment and simulation result by Bouc-Wen model.

the results are represented as a polynomial relationship. It is summarized in the Eq. (12)

$$
\begin{aligned}
& k=0.2875 \cdot V+0.3725 \\
& \gamma=1.1725 \cdot V+2.5071 \\
& n=0.0314 \cdot V+0.1391
\end{aligned}
$$

where $V$ is the amplitude value of the input voltage to the MFC, and $k, \gamma$, and $n$ are undetermined parameters in BoucWen model. Once the input voltages are confirmed, parameters $k, \gamma$, and $n$ would be obtained. When these parameters are plugged into the Bouc-Wen model, the induced strain $\varepsilon$ would be predicted. In order to verify the Bouc-Wen model is effective, a large number of reconstructed results are calculated based on the introduced cases in Section 2.2. As shown in Fig. 6, typical simulation results are compared with the experimental data in the case of $800 \mathrm{~V} / 400 \mathrm{~V}$ amplitude with the frequency of $0.5 \mathrm{~Hz} / 2 \mathrm{~Hz}$. It is found that the MFC's responses using the reconstructed Bouc-Wen model are in good agreement with the relative experimental data. After the results of different examples are collected, the errors maintain satisfactory accuracy level that is below $5 \%$ uniformly, which has laid a good foundation for the further research of active controller for MFC systems.

\section{DESIGN OF CONTROL LAW}

In this chapter, design of control law is introduced and applied to cantilever beams with MFC actuator. Based on the uncoupled motion equation of continuous systems, we deduce a time-delayed control equation where the active control force is related to a delayed feedback gain (control law). Here, the feedback gain is optimized by the GA according to various control objects such as vibration reduction, control energy etc.

\subsection{Vibration Control Equation for Cantilever Beam}

In Fig. 7, this paper mainly considers the transverse vibration of the cantilever beam excited by the MFC actuator. The beam has a constant cross-sectional area with every centre inertia axis in the same plane $x o z$. At the vicinal fixed end of the beam, there are two patches: one is MFC actuator used to control the vibration, and the other is MFC exciter used to stimulate the vibration.

Recently, more attention has been paid to the problems about unavoidable time-delay control. Based on the EulerBernoulli hypothesis, the time-delayed differential equation of motion for cantilever beam is given by ${ }^{19}$

$$
\begin{aligned}
E_{b} I_{b} \frac{\partial^{4} w(x, t)}{\partial x^{4}}+\rho \frac{\partial^{2} w(x, t)}{\partial x^{2}} & = \\
\sum_{m=1}^{M} H(x) \cdot f_{m}(x, t-\lambda) & +\sum_{n=1}^{N} H(x) \cdot q_{n}(x, t)
\end{aligned}
$$

where $E_{b}$ is elastic modulus of the beam material, $I_{b}$ represents section moment of inertia, and $\rho$ represents density of the material. Suppose control force derived from MFC actuators is distributed, then $f_{m}(x, t)$ represents the control force coming from the $m^{\text {th }}$ MFC actuator where $\lambda$ represents the time delay produced by the actuator. Likewise, $q_{n}(x, t)$ stands for the 


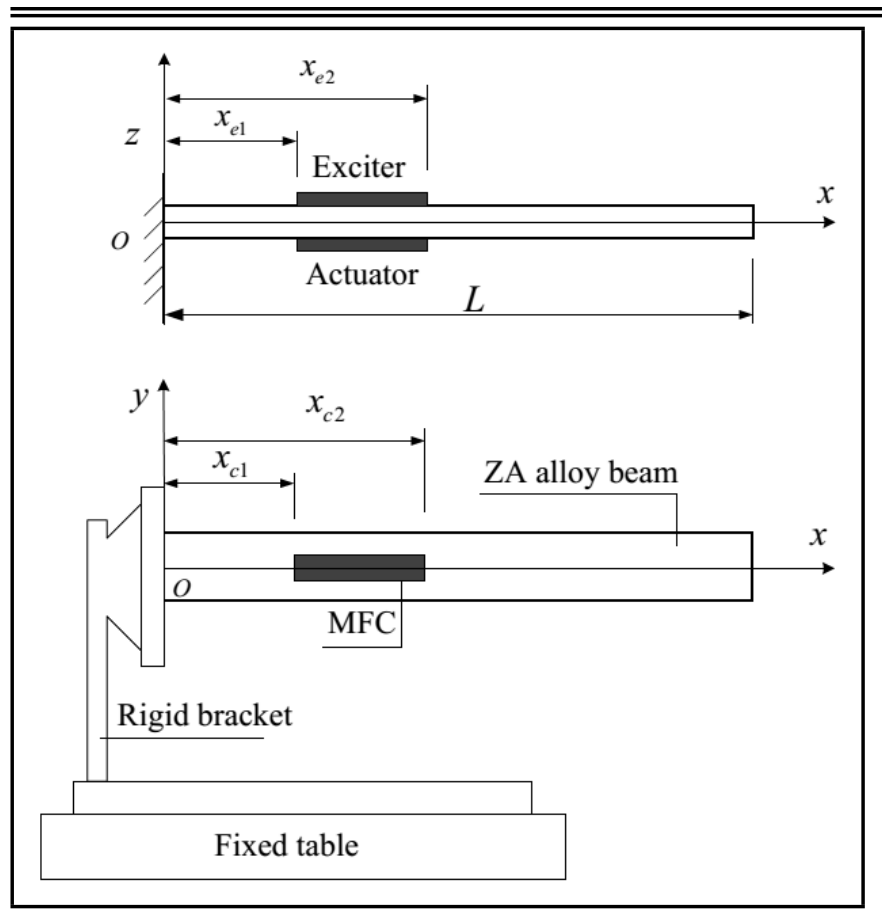

Figure 7. Cantilever beam model and the locations of MFC actuator.

measured strain from the $n^{\text {th }}$ exciter. $w(x, t)$ is transversal displacement of the beam at time $t$ in the location $x$ (referring to Fig. 7).

In Eq. (13), $H(x)$ is the threshold function. It is equal to $\delta\left(x-x_{1 n}\right)-\delta\left(x-x_{2 n}\right)$ where $x_{1 n}$ and $x_{2 n}$ denote the locations at the two ends of the $n^{\text {th }}$ MFC exciter. It can analogously be equal to $\delta\left(x-x_{1 m}\right)-\delta\left(x-x_{2 m}\right)$ where $x_{1 m}$ and $x_{2 m}$ denote the locations at the two ends of the $m^{\text {th }}$ MFC actuator. Here, $\delta(x)=\left\{\begin{array}{ll}1, & x \geq 0 \\ 0, & x<0\end{array}\right.$.

Using mode superposition method, we set $w(x, t)$ equal to $\sum_{i=1}^{n} \varphi_{i}(x) \eta_{i}(t)$ where $\eta_{i}$ is the $i^{\text {th }}$ modal coordinate, and $\varphi_{i}(x)$ is the normalized modal shape corresponding to the $i^{\text {th }}$ mode. In addition, it should be noted that the real vibration systems are always mitigated because of its inherent damping character. Hence, we also need to consider the damping factor in the controlled equation. In this sense, Eq. (13) can be decoupled as follows,

$\ddot{\eta}_{i}(t)+2 \zeta_{i} \omega_{i} \dot{\eta}_{i}(t)+\omega_{i}^{2} \eta_{i}(t)=$

$\int_{0}^{L} \varphi_{i}(x)\left[\sum_{m=1}^{M} H(x) \cdot f_{m}(x, t-\lambda)+\sum_{n=1}^{N} H(x) \cdot q_{n}(x, t)\right] d x$;

where $\omega_{i}$ is the natural frequency of the $i^{\text {th }}$ mode and $\zeta_{i}$ is the $i^{\text {th }}$ modal damping ratio.

In order to design a time-delay controller, modal equation should consider time delay in the control force. Suppose the first $n$-order modes are considered, then the modal equation involving one actuator and one exciter can be written as

$$
\ddot{\eta}(t)+C \dot{\eta}(t)+K \eta(t)=H_{c} U_{c}(t-\lambda)+H_{e} U_{e}(t) ;
$$

where $\eta(t)=\left[\begin{array}{c}\eta_{1}(t) \\ \eta_{2}(t) \\ \vdots \\ \eta_{n}(t)\end{array}\right], C=\left[\begin{array}{cccc}2 \zeta_{1} \omega_{1} & & & 0 \\ & 2 \zeta_{2} \omega_{2} & & \\ & & \ddots & \\ 0 & & & 2 \zeta_{n} \omega_{n}\end{array}\right]$, and $K=\left[\begin{array}{cccc}\omega_{1}^{2} & & & 0 \\ & \omega_{2}^{2} & & \\ & & \ddots & \\ 0 & & & \omega_{n}^{2}\end{array}\right] . U_{c}(t)$ is the applied voltage from the controller, in which $\lambda$ represents the time delay of control voltage produced by actuator. $U_{e}(t)$ is the excitation voltage from the exciter. $H_{c}$ is a transfer vector including information about actuator's position and its electric-mechanical behaviour. Likewise $H_{e}$ is another transfer vector including information of the exciter. In Section 4.3, the transfer vector will be introduced in detail.

In control engineering, the above dynamic equation is often cast as a set of first-order differential equations with time delay, using a state vector $Z=[\eta, \dot{\eta}]^{T}$, as follows:

$$
\dot{Z}(t)=A Z(t)+B U_{c}(t-\lambda)+D U_{e}(t)
$$

where $Z(t)$ is the $2 n$-dimensional state vector, $A$ is the $2 n \times 2 n$ system matrix, and $B$ and $D$ are the $2 n$-dimensional vectors. They are given by $Z(t)=\left[\begin{array}{l}\eta(t) \\ \dot{\eta}(t)\end{array}\right], A=\left[\begin{array}{cc}0 & I \\ -K & -C\end{array}\right], B=$ $\left[\begin{array}{c}0 \\ H_{c}\end{array}\right]$, and $D=\left[\begin{array}{c}0 \\ H_{e}\end{array}\right]$.

\subsection{Discretization and Standardization for Vibration Control Equation}

The solution of Eq. (16) is given by $\operatorname{Sun}^{21}$ as

$$
\begin{aligned}
Z(t)=e^{A\left(t-t_{0}\right)} Z\left(t_{0}\right)+ & \int_{t_{0}}^{t} e^{A(t-\tau)} B U_{c}(\tau-\lambda) d \tau+ \\
& \int_{t_{0}}^{t} e^{A(t-\tau)} D U_{e}(\tau) d \tau .
\end{aligned}
$$

The continuous-time form of the controlled equation is already presented; however, it also needs to be discretized and transformed into the standard discrete form for analysing a system on a digital computer or using digital controller. Thus, zero-order holder is used in the structure, i.e.

$$
Z(t)=Z(k T)
$$

where $Z(k T)$ is equivalent to the state of the system at time $t=k T$.

We may further simplify our notation by using $Z(k)$ instead of $Z(k T)$, and this notation proves convenient in mathematical manipulations. Meanwhile, time delay $\lambda$ should also be discretized and it is transferred into integral multiple of the sampling time $T$ that is

$$
\lambda=l T \text {. }
$$

According to the discretization of the continuous-time system, let $t_{0}=k T$ and $t=(k+1) T$, then Eq. (17) becomes

$$
\begin{aligned}
Z(k+1)=e^{A T} Z(k)+ & \int_{k T}^{(k+1) T} e^{A(k T+T-\tau)} B U_{c}(\tau-\lambda) d \tau+ \\
& \int_{k T}^{(k+1) T} e^{A(k T+T-\tau)} D U_{e}(\tau) d \tau . \quad \text { (20) }
\end{aligned}
$$




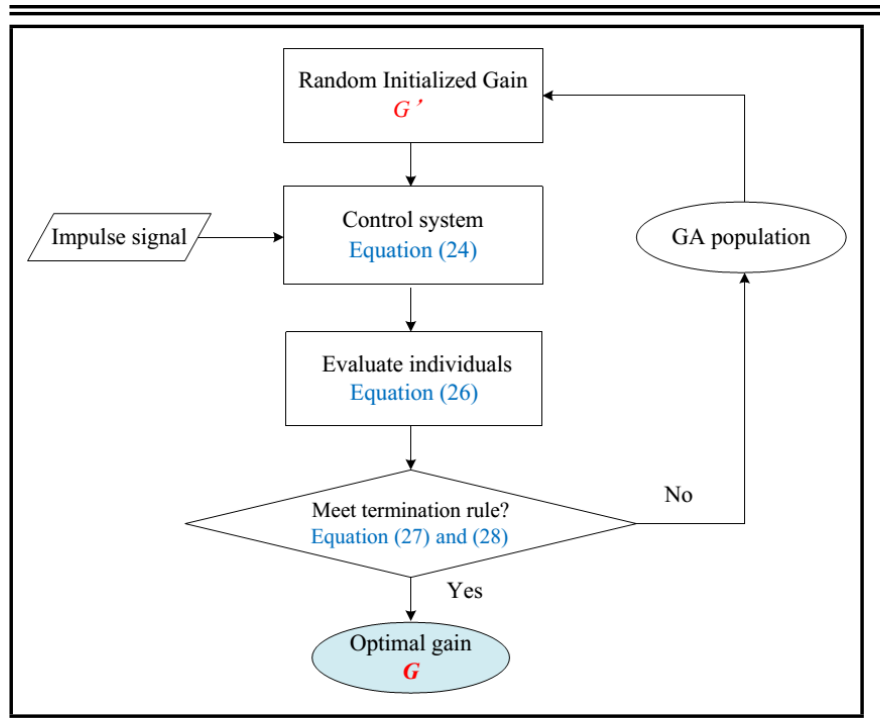

Figure 8. Flow chart of optimization of feedback control gain.

Making variable substitution $\eta=(k+1) T-\tau$, Eq. (20) becomes an iteration equation as

$$
\begin{aligned}
Z(k+1)=e^{A T} Z(k)+ & \int_{0}^{T} e^{A \eta} B U_{c}(k T+T-l T-\eta) d \eta+ \\
& \int_{0}^{T} e^{A \eta} D U_{e}(k T+T-\eta) d \eta
\end{aligned}
$$

Using Eq. (19) in Eq. (21), we get

$$
\begin{aligned}
Z(k+1) & =e^{A T} Z(k)+\int_{0}^{T} e^{A \eta} d \eta B U_{c}(k-l)+\int_{0}^{T} e^{A \eta} d \eta D U_{e}(k) \\
& =P Z(k)+R U_{c}(k-l)+E U_{e}(k) \\
R & =\int_{0}^{T} e^{A \eta} d \eta B, \quad E=\int_{0}^{T} e^{A \eta} d \eta D
\end{aligned}
$$

where $\mathrm{P}$ is $2 n \times 2 n$ matrix, and $R$ and $E$ are $2 n$-dimensional vectors.

To improve the dynamic performance of the controlled system, and because of inherent time delay in both controllers and actuators, the actual control force can be modelled as

$$
U_{c}(k-l)=-G Z(k-l) ;
$$

where $G$ is a $2 n$-dimensional vector, representing the feedback gains of response path. Then by casting Eq. (23) into Eq. (22) and without considering the external excitation, discretization and standardization of motion equation can be expressed by

$$
Z(k+1)=P Z(k)-R G Z(k-l) .
$$

\subsection{Optimization of Feedback Control Gain}

The inherent time delays in both controller and actuators albeit very short in most cases often deteriorate the control performance or even cause instability of the system. ${ }^{9,22}$ This paper aims to introduce a novel control algorithm based on the above motion equation for the systems with time delay.

Owing to the unpredictability of external excitation and unavoidability of time delay, it is found that a time-delayed system can be regulated by the control forces coming from the product of a static feedback gain and the time-delayed state (see Eq. (24)). Thus, we can use a parallel algorithm to search for an optimal feedback gain so as to mitigate the vibration response of structures at a certain degree. Additionally, based on modern control theory, ${ }^{23,24}$ the external excitation is usually neglected because of its unpredictability. Thus, this paper only considers the impulse signal to be the initial input. The flow chart of the GA controller is displayed in Fig. 8.

In this approach, the GA is designed in the following ways:

\section{(1) Chromosome structure}

In light of the nature characteristic of the problem to be solved, there are $2 n$ variables to be identified in Eq. (24). Thus, the chromosome structure becomes

$$
\Theta(k)_{i}=\{G\}=\left\{\left[g_{1}, g_{2}, \ldots, g_{j}, \ldots, g_{2 n}\right]\right\}, \quad i=1, \ldots, N \text {; }
$$

where $N$ is maximum number of chromosomes, $i / j$ represents the $i^{\text {th }} / j^{\text {th }}$ individual/variable in chromosome, and $n$ represents the total number of modals we will consider.

\section{(2) Objective function}

An objective function is a measuring mechanism that is used to evaluate the status of every chromosome (individual) within a population. This is a very important link to relate the GA and system concerned. Since each chromosome is individually going through the same evaluating exercise, the range of this value varies from one chromosome to another. In order to maintain uniformity, the objective value $O b j$ is mapped into a fitness value with a map $\Psi$ where the domain of $F i t$ is usually greater than zero and moderate value. According to the described problem in this study, this paper designs modal coordinate as the objective function

$$
O b j=\alpha \sum_{k=1}^{K}\left|\eta_{1}(k)\right|+\beta \sum_{k=1}^{K}\left|\eta_{2}(k)\right|+\cdots+\gamma \sum_{k=1}^{K}\left|\eta_{n}(k)\right|
$$

where $\eta_{1}(k), \eta_{2}(k)$, and $\eta_{n}(k)$ are the $1^{\text {st }}, 2^{\text {nd }}$, and $n^{\text {th }}$ modal coordinate in the $k^{\text {th }}$ data point whose total number is $K$. The weighting coefficients $\alpha, \beta, \ldots, \gamma$ reflect the relative importance of the corresponding modal. For example, if the first two modals are considered with weighting coefficients $\alpha=1$ and $\beta=0$, GA controller can realize the optimal vibration absorption for the first modal.

\section{(3) Termination rule}

Early GAs generally terminate after the expiry of a fixed number of genetic generations. However, when the solution has already found the minimum error but not yet arrived the designated generation, it is undoubtedly wasteful to go on iterating. For this reason, an appropriate termination rule should be created so as to relive unnecessary computational burden.

The termination strategy in this study is proposed under two aspects: one is depending on maturity degree of the population proceeding in a certain generation. Another is in view of the fittest individuals after iterating a certain generation, controlling the real calculation accuracy.

Rule 1: Terminate the GA if a reduction of minimum error (that is equal to the value of the objective) lower than $20 \%$ of maximum error does not arise in further generation, which is defined as:

$$
O b j_{\min }^{k}-O b j_{\min }^{k+1} \leq 0.2 \max _{k} O b j_{\min }^{k}
$$




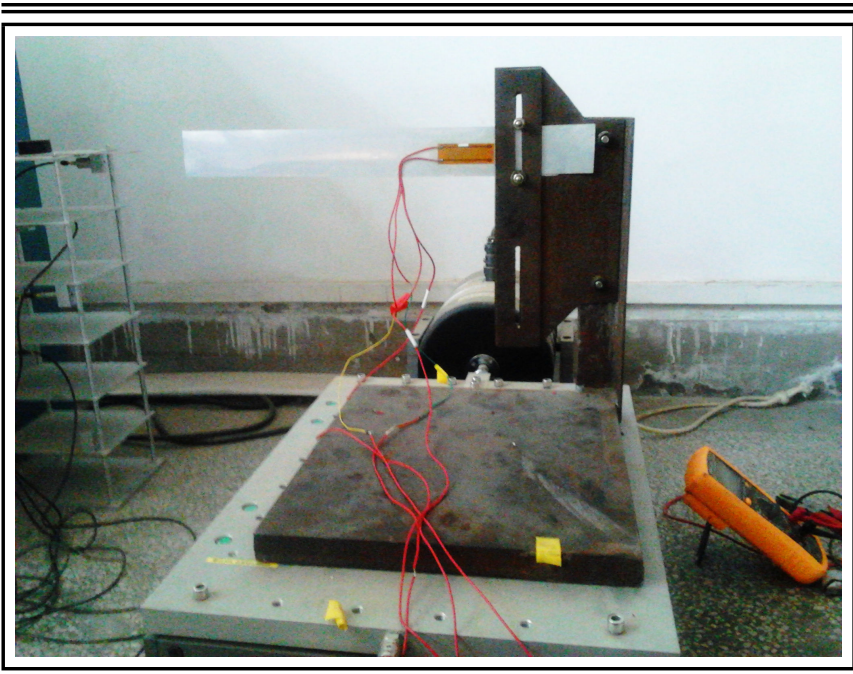

Figure 9. Vibration control platform of cantilever beam with MFC actuator.

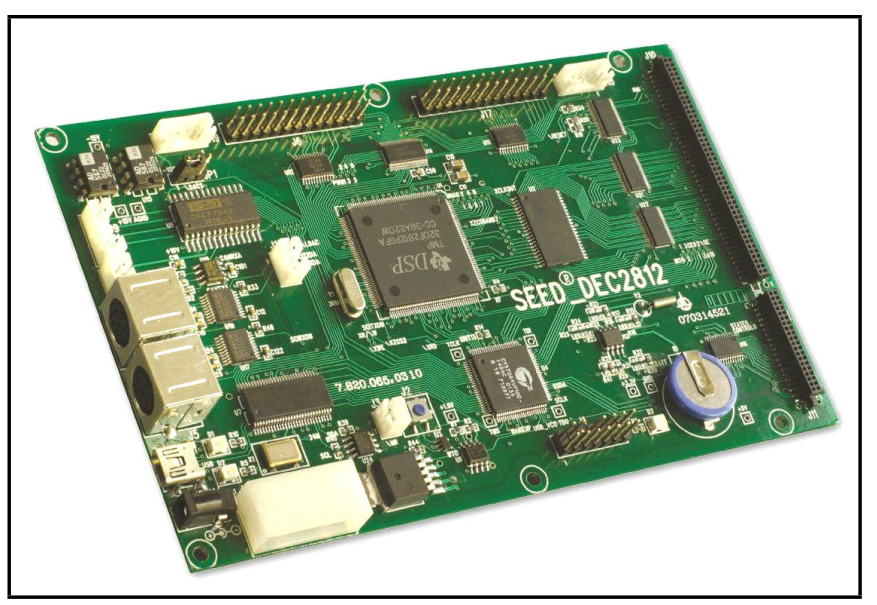

Figure 10. SEED DEC2812 DSP.

where $O b j_{\min }^{k}$ represents the minimum value of objective function in $k^{\text {th }}$ generation. This rule can guarantee that the population should not be diversified any more, and the best individual is very close to the global solution.

Rule 2: Terminate the GA if the minimum objective value is below a threshold $O_{\min }$, which is given by

$$
O b j_{\min }^{k} \leq O_{\min }
$$

where $O_{\min }$ is the designate threshold value of objective. This rule can guarantee that the final solution's precision is adequately satisfied. The rest algorithm settings are set as follows: roulette wheel selection, crossover rate $P_{c}=0.85$, mutation rate $P_{m}=0.01$.

\section{EXPERIMENT OF CANTILEVER BEAM CONTROLLED BY MFC ACTUATOR}

This section introduces a vibration test platform of cantilever beam restricted by active control system using MFC actuator. Figure 9 displays the real test platform of cantilever beam controlled system.

\subsection{Experiment Setup}

In the test platform, one MFC patch is used as actuator, two strain gauges as sensors, and DSP as controller to realize the control algorithm. The experiment setup is constructed using

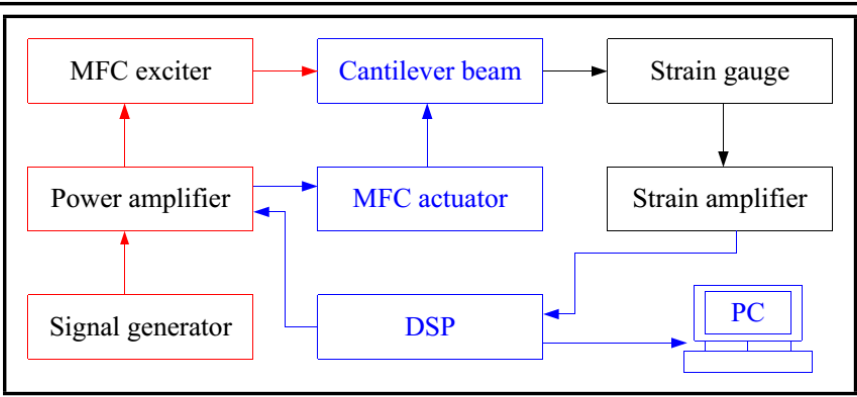

Figure 11. Flow chart of vibration test.

SEED DEC2812 DSP (see Fig. 10). The DSP deals with online computation of controller in terms of feedback signal from the strain gauge to obtain MFC voltage. Here, the beam is made of lead-aluminium alloy, whose length, width, and thickness are $350 \mathrm{~mm}, 20 \mathrm{~mm}$, and $0.2 \mathrm{~mm}$, respectively. Material properties of the beam are as follows: Young's elastic modulus $E=80 \mathrm{GPa}$, Poisson's ratio $\nu=0.3$, and density $\rho=7.1 \times 10^{3} \mathrm{~kg} / \mathrm{m}^{3}$. The first two natural frequencies of the beam determined from the experiment are $0.5252 \mathrm{~Hz}$ and $3.1831 \mathrm{~Hz}$ respectively, and the corresponding modal damping ratios are set as 0.02 .

Figure 11 shows an experimental flow chart of a flexible cantilever beam excited by an MFC exciter and then controlled by an MFC actuator. It is described in three parts:

(1) Excitation part: signal generator generates an external excitation that is amplified by the power amplifier and then goes into the MFC exciter. This part is illustrated with red block diagrams in Fig. 11.

(2) Measuring signal part: the signal collected from the strain gauge is amplified by a strain signal amplifier and then enters in to DSP. This part is illustrated with black rectangles in Fig. 11.

(3) Control part: $\quad$ DSP controller receives the response signal collected from the strain gauges. Through a series of calculation using GA control algorithm, DSP controller delivers the calculated voltage control signal into power amplifier, and then the amplified signal is channelled into the MFC actuator. This part is illustrated with blue rectangles in Fig. 11.

\subsection{Signal Transform for Sensor}

The controller described in the preceding section depends on the beam modal coordinate. So a method to extract modal coordinate from the physical sensor measurements of strain gauges needs to be developed.

The beam strain-curvature relationship can be given by

$$
\varepsilon(x, t)=\frac{t_{b}}{2} w^{\prime \prime}(x, t)
$$

where $\varepsilon, t_{b}$, and $w$ are separately the bending strain, thickness, and the transverse displacement of the beam.

Using mode superposition method, the transverse displacement $w(x, t)$ is decoupled by $\sum_{i=1}^{n} \varphi_{i}(x) \eta_{i}(t)$ (see Section 3.1). On the basis of the modal cost analysis method, ${ }^{25}$ the first two order modals are dominant, which are considered in this paper. 


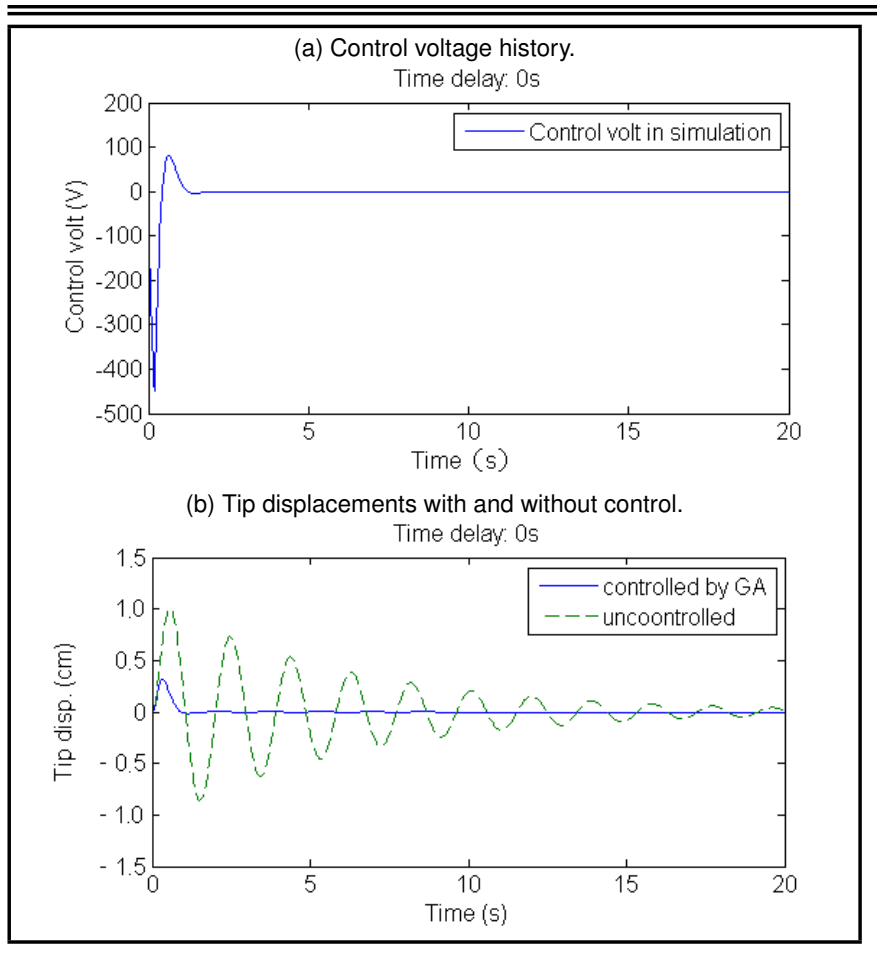

Figure 12. Simulation results using GA controller with $0 \mathrm{~s}$ time delay under transient excitation.

Hence, the transverse displacement responses of the beam can be deduced by vibration model function and measured strains from experimental test. It is given by

$w(x, t)=\frac{2}{t_{b}}\left[\begin{array}{ll}\varphi_{1}(x) & \varphi_{2}(x)\end{array}\right]\left[\begin{array}{cc}\varphi_{1}^{\prime \prime}\left(x_{s 1}\right) & \varphi_{2}^{\prime \prime}\left(x_{s 1}\right) \\ \varphi_{1}^{\prime \prime}\left(x_{s 2}\right) & \varphi_{2}^{\prime \prime}\left(x_{s 2}\right)\end{array}\right]\left[\begin{array}{l}\varepsilon\left(x_{s 1}, t\right) \\ \varepsilon\left(x_{s 2}, t\right)\end{array}\right] ;$

where $w(x, t)$ is the transverse displacement at time $t$ in position $x$ under the coordinates in Fig. 7, $\varphi_{i}(x)$ is the $i^{\text {th }}$ order vibration mode function, $\varepsilon\left(x_{s i}, t\right)$ stands for the measured strains at time $t$, and $x_{s i}$ is the distance from the fixed end of the cantilever beam to the location of the $i^{\text {th }}$ strain gauge.

\subsection{Signal Transform for Actuator}

In addition to deducing the relationship between modal coordinate and measured strain data from sensors, extraction of modal coordinate from the MFC actuator also needs to be developed. As soon as the MFC patch is pasted on the beam, the connection between the MFC actuator and the beam results in mechanical electric coupling. Hence, the constrained strain $\varepsilon_{0}$ would be magnified from the free strain $\varepsilon$ (referring to previous work $^{7}$ ) that is

$$
\varepsilon_{0}=\frac{12}{6+E_{b} h_{b} / E_{m} h_{m}} \cdot \varepsilon ;
$$

where $E_{b} / E_{m}$ is Young's modulus of the beam/MFC, and $h_{b} / h_{m}$ is the width of the beam/MFC.

Due to the consistency principle, the stresses at the same point between the beam and the MFC are equal to each other, provided that these two components cohere very well. Upon that, the stress at the cross section is given by Eq. (32), based on the character of Euler-Bernoulli beam,

$$
\sigma=\frac{\sigma_{0}}{h_{b}} y
$$

where $y$ is the vertical distance between neutral axis and stress point at the cross section of the beam. (a) Control voltage history.

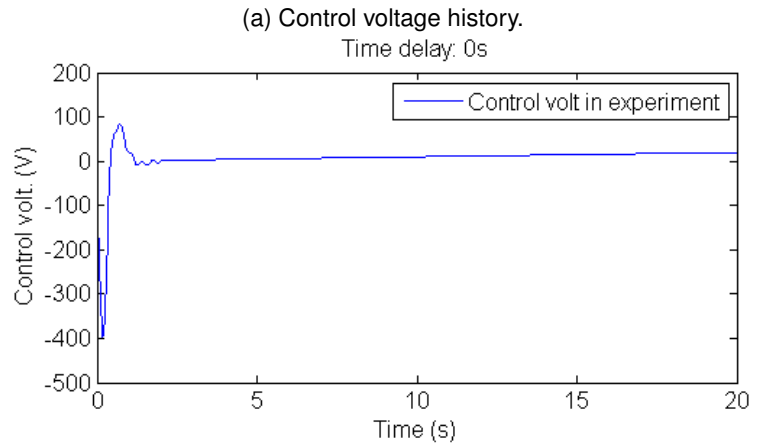

(b) Tip displacements with and without control. Time delay. Os

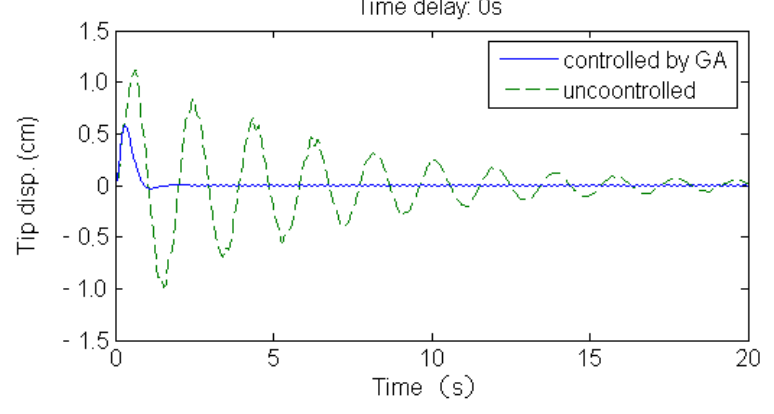

Figure 13. Experiment results using GA controller with $0 \mathrm{~s}$ time delay under transient excitation.

Based on mechanics theory, the MFC can provide bending moment to the beam that is

$$
M(x, t)=\int_{A} \sigma y d A=\frac{\sigma_{0}}{h_{b}} \cdot I_{z}
$$

Substituting Eqs. (31) and (32) into Eq. (33), we get

$$
M(x, t)=\frac{12 E_{b} I_{z}}{\left(6+E_{b} h_{b} / E_{m} h_{m}\right) h_{b}} \cdot \varepsilon ;
$$

where $\frac{12 E_{b} I_{z}}{\left(6+E_{b} h_{b} / E_{m} h_{m}\right) h_{b}}$ is constant coefficient relating to the inherent characteristic of the controlled systems, and the constrained strain $\varepsilon$ is an important variable derived from the Bouc-Wen model illustrated in Section 2.3. Here, it is very important to note that control voltage is one latent variable existed in Bouc-Wen model. If the voltage is calculated directly, an abnormal solution will be often obtained such as complex root. Therefore, obtaining an effective solution is important. In this paper, we can bypass the traditional method, but extract the effective inverse voltage by using the GA. Similar to the design of control law, this GA is set as follows.

\section{(1) Chromosome structure}

$$
\Theta(k)=\{\text { Volt }\}, \quad k=1, \ldots, N
$$

where $N$ is maximum number of chromosomes, $k$ is the $k^{\text {th }}$ individual in chromosome, and Volt is the unknown variable (control voltage), and it can be optimized or calculated by using a GA procedure. We set the value of the Volt within the range between $-600 \mathrm{~V}$ and $600 \mathrm{~V}$.

\section{(2) Objective function}

$$
O b j=\left(f_{c}^{\prime}-f_{c}\right)^{2}
$$

where $f_{c}$ represents the optimal control force according to the calculated control law from the GA controller (referring to 


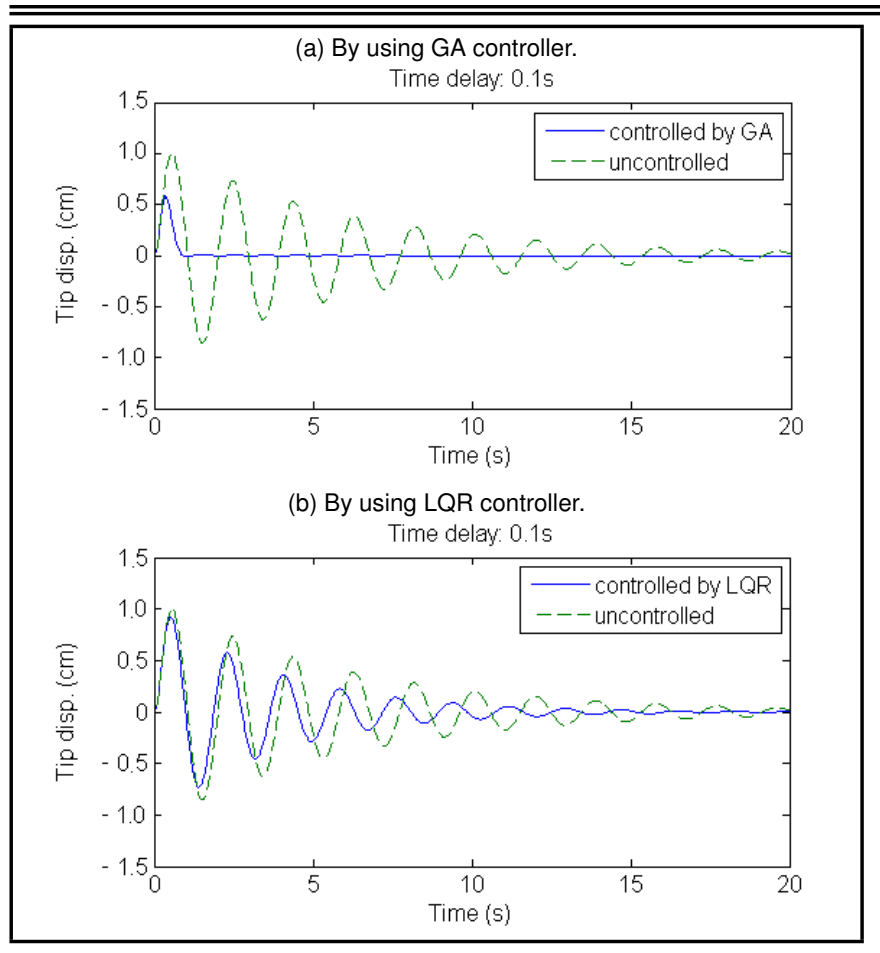

Figure 14. Simulation results using different controller with $0.1 \mathrm{~s}$ time delay under transient excitation.

Section 3), and $f_{c}^{\prime}$ is the control force by assigning the variable Volt to the Bouc-Wen model. After a handful of iterations, the effective control voltage will be extracted consequently.

\section{ANALYSIS FOR EXPERIMENTAL AND NUMERICAL RESULTS}

To demonstrate the application of the proposed method and its performance, experimental and numerical results of the cantilever beam controlled by an MFC actuator are illustrated in this section. Note that the MFC actuator and exciter are pasted near the fixed end of the beam where $x_{1}=25 \mathrm{~mm}$ and $x_{2}=85 \mathrm{~mm}$ referring to Fig. 7 . In the experiment, two types of external excitations are considered, one of which is transient signal input, and the other is random signal input. Then the simulation results under the same excitations are calculated for easy comparative analysis with experimental results.

In general, the first modal in common systems basically dominates the whole vibration energy. Therefore, this paper set weighting coefficients as $\alpha=1$ and $\beta=0$ to mainly mitigate the first modal vibration.

\subsection{Transient Excitation}

In the experiment, the signal generator generates a transient signal with voltage amplitude $500 \mathrm{~V}$ in order to motivate beam vibrations. The MFC actuator under the instruction of the GA controller is used for controlling the vibration. Let us first focus on the simulation results without time delay. When control voltage is applied to MFC in Fig. 12(a), the beam is distinctly suppressed, whose tip peak displacement $10.2 \mathrm{~mm}$ is great reduced to $2.6 \mathrm{~mm}$ using the GA controller (see Fig. 12(b)). Apparently the simulation result is satisfactory through the proposed approach. Next, noting the experimental result, the beam is also obviously suppressed, whose tip peak displacement $11 \mathrm{~mm}$ is significantly decreased to $5.7 \mathrm{~mm}$

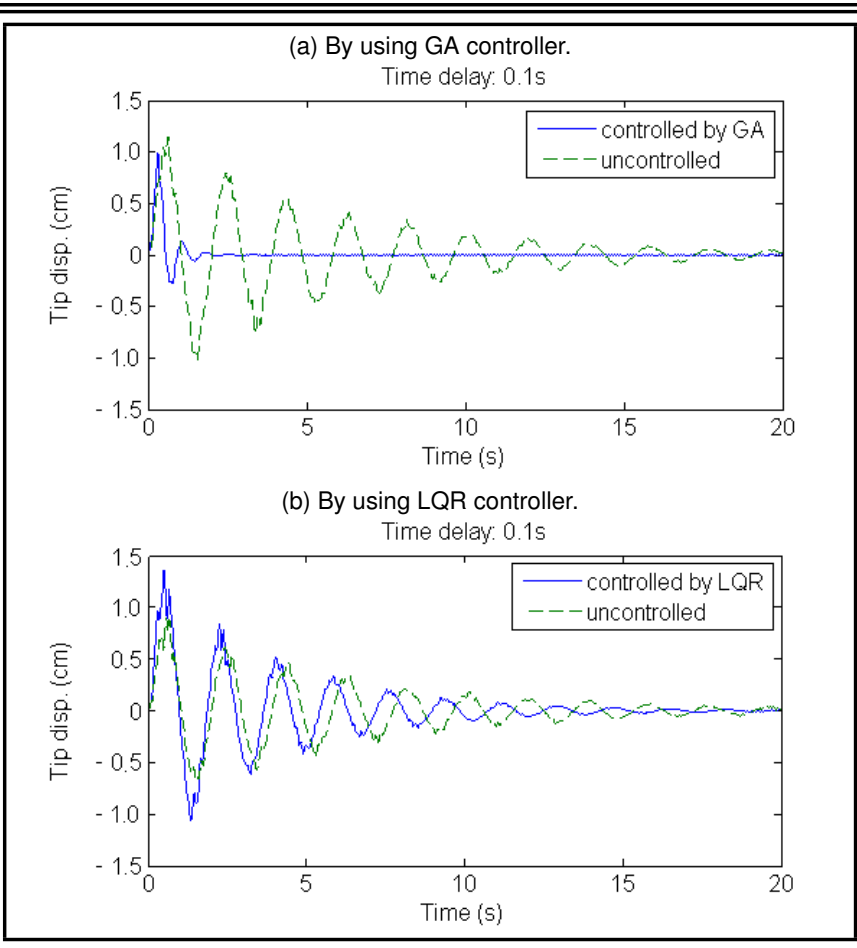

Figure 15. Experiment results using different controller with $0.1 \mathrm{~s}$ time delay under transient excitation.

Table 2. Comparison between the method $L Q R$ and GA under transient excitation.

\begin{tabular}{|c|c|c|c|c|c|}
\hline \multicolumn{6}{|c|}{ Beam tip disp. reduction (\%) / Control voltage peak (V) } \\
\hline & & $0 \mathrm{~s}$ & $0.01 \mathrm{~s}$ & $0.02 \mathrm{~s}$ & $0.2 \mathrm{~s}$ \\
\hline \multirow{2}{*}{ GA } & Simulation & $70 / 450$ & $65 / 450$ & $62 / 450$ & $45 / 450$ \\
\hline & Experiment & $50 / 396$ & $49 / 391$ & $48 / 385$ & $21 / 366$ \\
\hline \multirow{2}{*}{ LQR } & Simulation & $70 / 450$ & $22 / 165$ & $10 / 100$ & Control failure \\
\hline & Experiment & $50 / 396$ & $12 / 75$ & $5 / 68$ & Control failure \\
\hline
\end{tabular}

Table 3. Comparison between the method LQR and GA under random excitation.

\begin{tabular}{|c|c|c|c|c|c|}
\hline \multicolumn{6}{|c|}{ Beam tip disp. reduction (\%) / Control voltage peak (V) } \\
\hline \multirow{2}{*}{ GA } & Simulation & $0 \mathrm{~s}$ & $0.01 \mathrm{~s}$ & $0.02 \mathrm{~s}$ & $0.2 \mathrm{~s}$ \\
& Experiment & $45 / 357$ & $75 / 251$ & $43 / 238$ & $37 / 222$ \\
& Simulation & $77 / 355$ & $24 / 126$ & Control failure & Control failure \\
\multirow{2}{*}{ LQR } & Experiment & $35 / 301$ & $10 / 86$ & Control failure & Control failure \\
& &
\end{tabular}

(see Fig. 13(b)), when control voltage is applied to MFC actuator in Fig. 13(a).

For comparing and verifying the superiority of the GA controller, classical linear quadratic optimal control (LQR) is also employed in this section. It is observed that the weighting matrices $Q$ and $R$ are chosen according to the "weight", i.e., according to the importance we want to assign to each element of the state vector and the input vector. For the present example, we have $Q=\operatorname{diag}[10000,10000,1,1]$ and $R=\left[10^{-10}\right]$. Through comparison between LQR and GA controller in Table 2 , it is found that the beam vibration can be suppressed effectively by using the GA controller, and the proposed controller is also applicable to different time delays. On the contrary, the LQR does not have a very obvious damping effect consistently. When the time delay appears, the vibration reduction becomes lower until control failure occurs. Figure 14 illustrates that the vibration reduction effect in simulating calculations is much better by using time-delayed GA controller than by LQR method. Likewise, experiment results in Fig. 15 also show that GA controller is superior to the LQR, especially when time delay appears. 


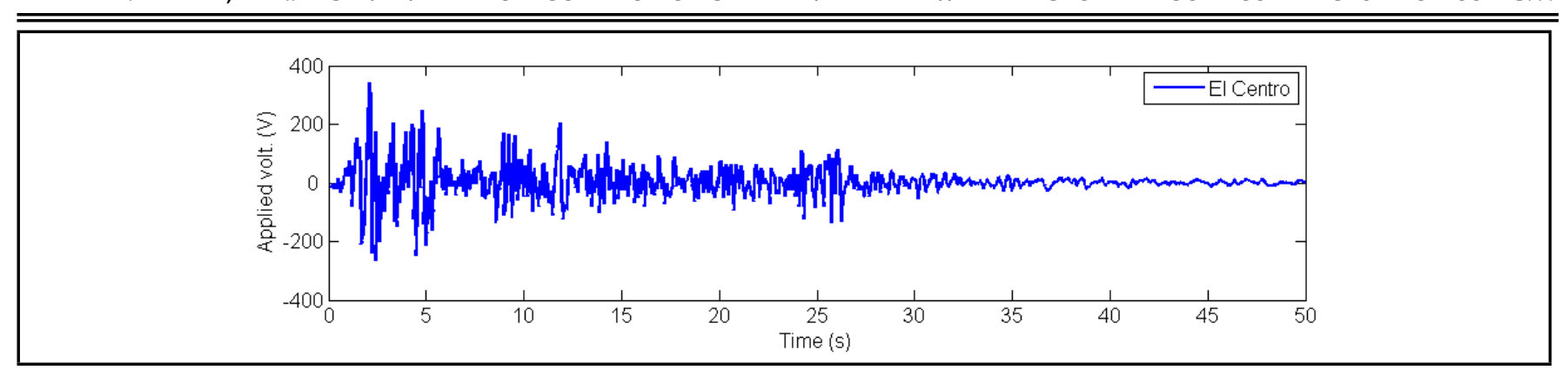

Figure 16. Applied voltages based on EI Centro record.

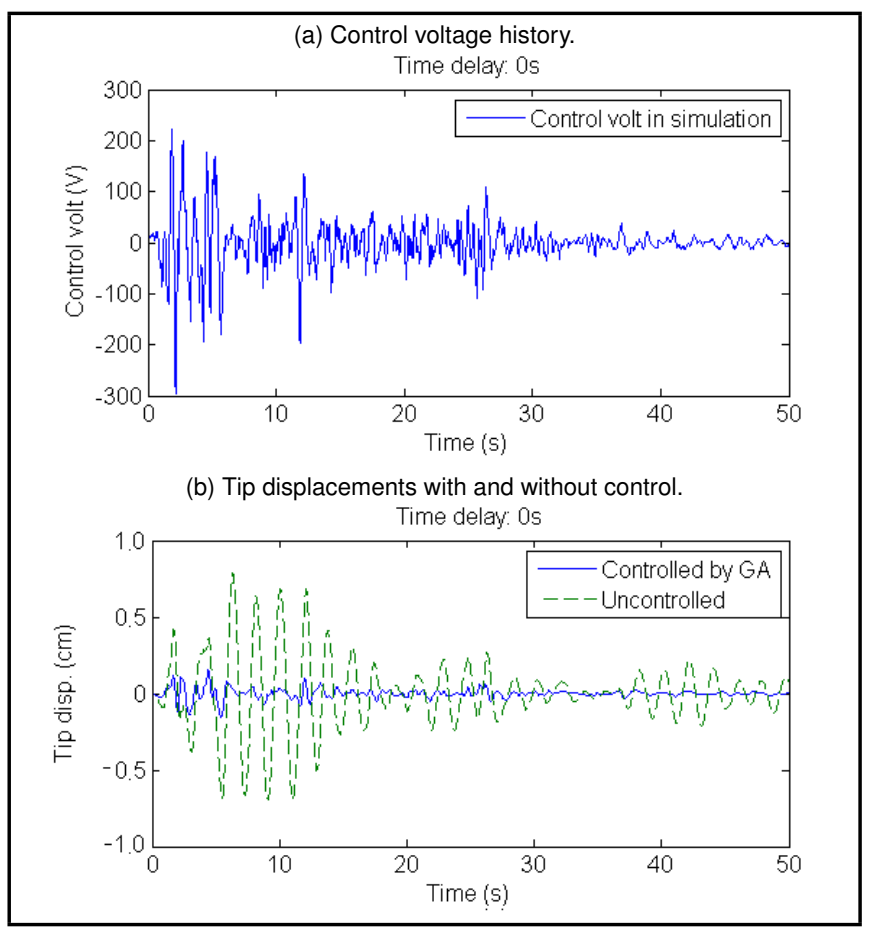

Figure 17. Simulation results using GA controller with $0 \mathrm{~s}$ time delay under random excitation.

\subsection{Random Excitation}

Further examples for the control of the beam vibration are presented to demonstrate the effectiveness of the proposed GA controller. This paper adopts the NS components of EI Centro acceleration record as external excitation. In order to excite and control the system within the rated voltage range, the seismic acceleration amplitude is adjusted to equivalent voltage record through power amplifier in Fig. 16. Consequently, a series of simulation and test under random excitation are carried out as following.

The control voltage is calculated by the GA controller in Fig. 17(a), and then applied to the beam. We found the beam is distinctly suppressed, whose tip peak displacement $7.8 \mathrm{~mm}$ is significantly reduced to $1.6 \mathrm{~mm}$ in Fig. 17(b). Note the experiment results in Fig. 18; the beam is still effectively suppressed, whose tip peak displacement $6.7 \mathrm{~mm}$ is decreased to $3.7 \mathrm{~mm}$.

Next the time-delay controller is also investigated under random excitation. When time delay is set as $0.2 \mathrm{~s}$, the results using a time-delay controller for controlling beam vibration are shown in Fig. 19 and Fig. 20 that are, respectively, from simulation and experiment. It is analogous to the non-time-delayed one, where the reduction of tip response is also effective by GA controller.

We have also obtained the results from LQR method in

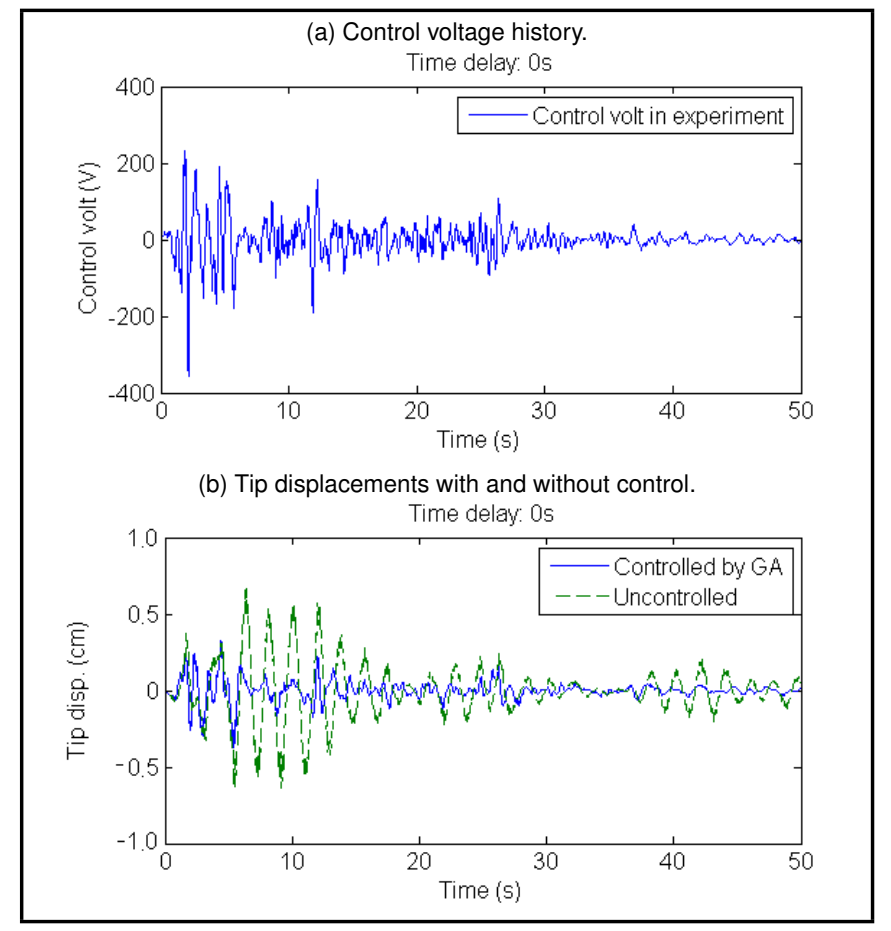

Figure 18. Experiment results using GA controller with $0 \mathrm{~s}$ time delay under random excitation.

Fig. 21. Comparing the results from the GA controller with LQR method, GA controller is found more effective in reducing the structural response, particularly in the condition of different time delays, that is always the most difficult to be reduced in active control structures by using classic algorithm such as LQR method. In addition, note an interesting phenomenon in Fig. 21; when method LQR is used with the time delay $0.2 \mathrm{~s}$, the simulation results illustrate that the responses obviously diverge, but the experiment results show that the responses with control and without control are analogous. This interesting phenomenon is because of the diverging responses that are often restricted by the actual test conditions. In general, the GA controller is able to control the beam vibration effectively and the experiment results agree better with respect to simulation in Table 3 .

Through the simulation and experimental results from the different examples, some general conclusions are drawn as follows.

\section{(1) Time delay vs. vibration reduction}

As illustrated in Table 2 and Table 3, structural vibration can be mitigated by these two control methods without time delay. Once time delay exists in the system and is increased from $0 \mathrm{~s}$ to a certain value, the control effect by LQR method will be worse and worse until failure control. Unlike LQR, the GA 


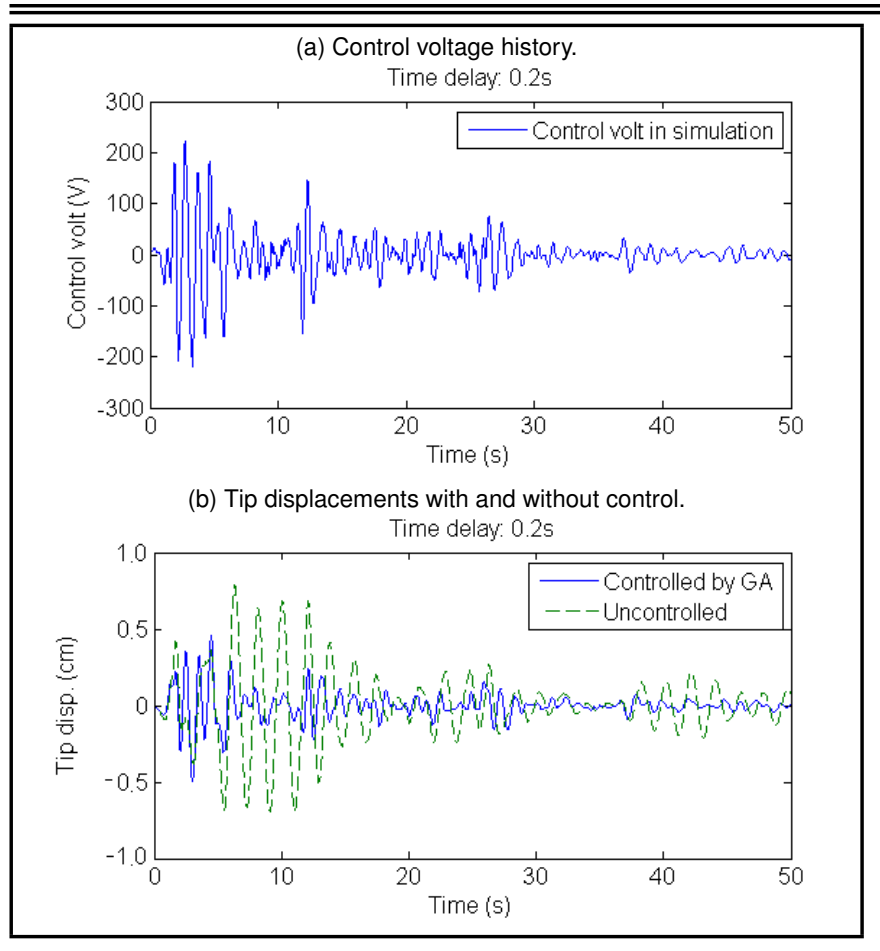

Figure 19. Simulation results using GA controller with $0.2 \mathrm{~s}$ time delay under random excitation.

controller can obtain a better control effect and also guarantee effective reduction even with a relative large time delay $(0.2 \mathrm{~s})$.

\section{(2) Control voltage vs. vibration reduction}

In GA controller design, the optimized control gain is not invariable, but mainly dependent on the optimizing range of the control voltage that generally results in a larger voltage for controlling the structural vibration. By contrasting and analysing in experiment and simulation, we found that the increasing range of control voltage will lead to relative better damping effect in controlled systems.

\section{(3) Time delay vs. control voltage}

It is generally known that time delay can cause worse or even adverse effects on the control action. Beyond that, the optimizing range of control voltage in GA can also affect its control performance involving different time delays. When the range of control voltage is set as a larger value, time delay will be more sensitive to the system's stability, which may more easily give rise to control failure.

Based on the above phenomenon, time delay, control voltage, and vibration reduction are closely related. In order to obtain satisfactory reduction effect in structural control systems, we should consider these factors altogether.

\section{CONCLUSIONS}

In this paper, an active control algorithm using GA is proposed for mitigating a cantilever beam actuated by an MFC patch. First, an electric-mechanical behaviour of the MFC is investigated through experiment, and a mathematic relation between applied voltage and relative strain is presented in preparation for control algorithm design. Second, an optimized time-delayed control method is proposed by a GA procedure in order to mitigate one time-delayed continuous system. Finally, the numerical and experimental results from a cantilever (a) Control voltage history.

Time delay: $0.2 \mathrm{~s}$

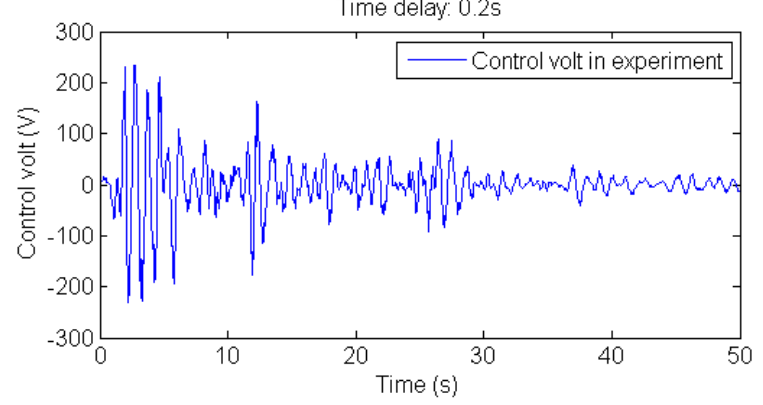

(b) Tip displacements with and without control. Time delay : $0.2 \mathrm{~s}$

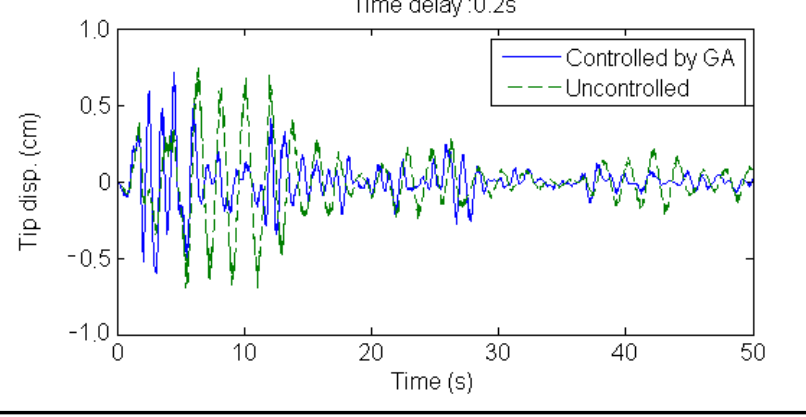

Figure 20. Experiment results using GA controller with $0.2 \mathrm{~s}$ time delay under random excitation.

beam controlled system are utilized to testify that the proposed approaches are effective in controlling beam vibration with different time delays. This study will also throw light on the development and control of other novel structures.

\section{ACKNOWLEDGMENTS}

This research is financially supported by the project from the National Natural Science Foundation, People's Republic of China, grants No.11172226 and No.11502188. The supports are gratefully acknowledged.

\section{REFERENCES}

1 Kwak, M. K. and Heo S. Active vibration control of smart grid structure by multiinput and multioutput positive position feedback controller, Journal of Sound and Vibration, 304 (1), 230-245, (2007). https://dx.doi.org/10.1016/j.jsv.2007.02.021

2 Gibson, R. F. A review of recent research on mechanics of multifunctional composite materials and structures, Composite Structures, 92 (12), 2793-2810, (2010). https://dx.doi.org/10.1016/j.compstruct.2010.05.003

3 Khot, S. M., Yelve, N. P., Tomar, R., Desai, S., and Vittal, S. Active vibration control of cantilever beam by using PID based output feedback controller, Journal of Vibration and Control, 18 (3), 366-372, (2012). https://dx.doi.org/10.1177/1077546311406307

${ }^{4} \mathrm{Ge}, \mathrm{P}$. and Jouaneh, M. Modeling hysteresis in piezoceramic actuators, Precision Engineering, 17 (3), 211-221, (1995). https://dx.doi.org/10.1016/0141-6359(95)00002-u

5 Arlt, K. and Wegener, M. Piezoelectric PZT/PVDFcopolymer 0-3 composites: Aspects on film preparation and electrical poling, IEEE Transactions on Dielectrics 


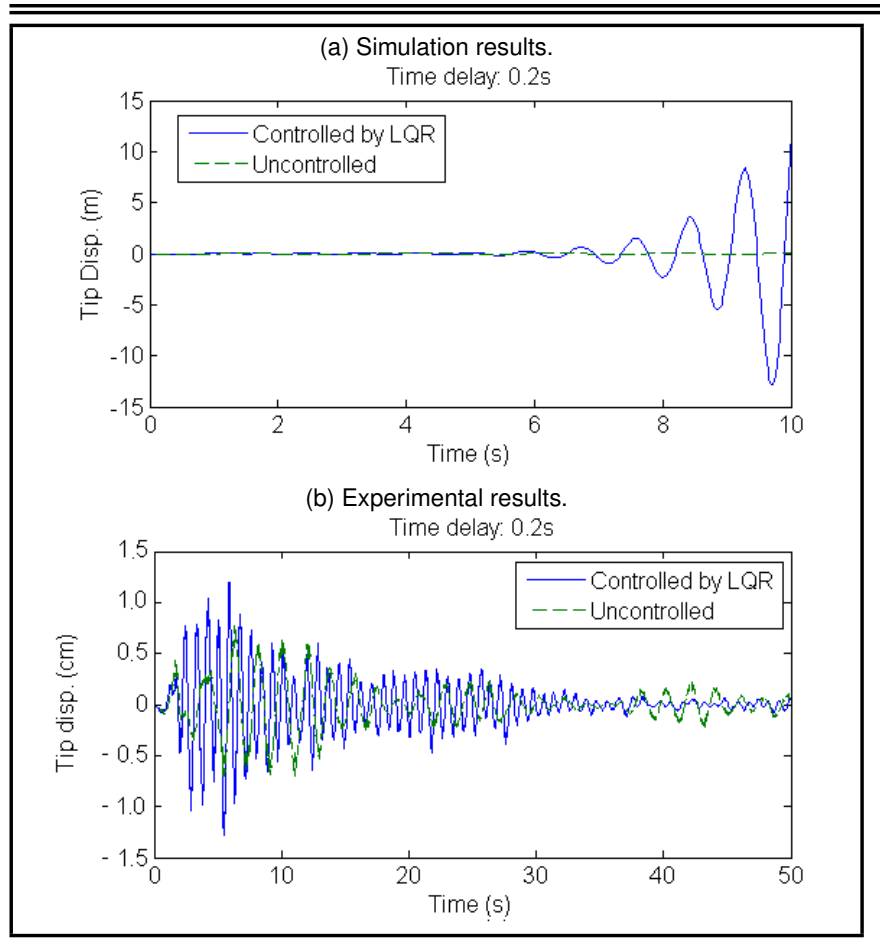

Figure 21. Results by using LQR controller with $0.2 \mathrm{~s}$ time delay under random excitation.

and Electrical Insulation, 17 (4), 1178-1184, (2010). https://dx.doi.org/10.1109/tdei.2010.5539688

6 Sodano, H. A., Park, G., and Inman, D. J. An investigation into the performance of macro-fiber composites for sensing and structural vibration applications, Mechanical Systems and Signal Processing, 18 (3), 683-697, (2004). https://dx.doi.org/10.1016/s0888-3270(03)00081-5

7 Deraemaeker, A. and Nasser, H. Numerical evaluation of the equivalent properties of Macro Fiber Composite (MFC) transducers using periodic homogenization, International Journal of Solids and Structures, 47 (24), 3272-3285, (2010). https://dx.doi.org/10.1016/j.ijsolstr.2010.08.006

8 Wilkie, W. K., Bryant, R. G., High, J. W., Fox, R. L., Hellbaum, R. F., Jalink Jr, A., and Mirick, P. H. Low-cost piezocomposite actuator for structural control applications, In SPIE's 7th Annual International Symposium on Smart Structures and Materials, International Society for Optics and Photonics, 323-334, (2000). https://dx.doi.org/10.1117/12.388175

$9 \mathrm{Hu}, \mathrm{H}$. Y. and Wang, Z. Dynamics of controlled mechanical systems with delayed feedback, Springer, (2002). https://dx.doi.org/10.1007/978-3-662-05030-9

10 Abdel-Rohman, M. Time-delay effects on actively damped structures, Journal of Engineering Mechanics, 113 (11), 1709-1719, (1987). https://dx.doi.org/10.1061/(asce)07339399(1987)113:11(1709)

11 Abdel-Mooty, M. and Roorda, J. Time-delay compensation in active damping of structures, Journal of Engineering Mechanics, 117 (11), 25492570, (1991). https://dx.doi.org/10.1061/(asce)07339399(1991)117:11(2549)
12 Abdel-Rohman, M., John, M. J., and Hassan, M. F. Compensation of time delay effect in semiactive controlled suspension bridges, Journal of $\mathrm{Vi}$ bration and Control, 16 (10), 1527-1558, (2010). https://dx.doi.org/10.1177/1077546309106518

13 Shao, M. Q. and Chen, W. D. Active vibration control in a cantilever-like structure: a time delay compensation approach, Journal of Vibration and Control, 19 (5), 674-685, (2013). https://dx.doi.org/10.1177/1077546312437802

14 Niu, Y., Ho, D. W. C., and Lam, J. Robust integral sliding mode control for uncertain stochastic systems with time-varying delay, Automatica, 41 (5), 873-880, (2005). https://dx.doi.org/10.1016/j.automatica.2004.11.035

15 Wang, B., Shi, P., Karimi, H. R., and Wang, J. HInfinity robust controller design for the synchronization of master-slave chaotic systems with disturbance input, Modeling, Identification and Control, 33 (1), 27-34, (2012). https://dx.doi.org/10.4173/mic.2012.1.3

16 Guoping, C. and Jinzhi, H. Optimal control method for seismically excited building structures with time-delay in control, Journal of Engineering Mechanics, 128 (6), 602-612, (2002). https://dx.doi.org/10.1061/(asce)07339399(2002)128:6(602)

17 Pan, I., Das, S., and Gupta, A. Tuning of an optimal fuzzy PID controller with stochastic algorithms for networked control systems with random time delay, ISA Transactions, 50 (1), 28-36, (2011). https://dx.doi.org/10.1016/j.isatra.2010.10.005

18 Williams, R. B. Nonlinear mechanical and actuation characterization of piezoceramic fiber composites, Diss. Virginia Polytechnic Institute and State University, (2004). https://dx.doi.org/10.18297/etd/855

19 Bishop, R. E. D. and Johnson, D. C. The mechanics of vibration, Cambridge University Press, (2011).

20 Xue, X. M., Chen, L. Q., Wu, X. H., and Sun, Q. S. Study on electric-mechanical hysteretic model of MacroFiber Composite actuator, Journal of Intelligent Material Systems and Structures, 25 (12), 1469-1483, (2013). https://dx.doi.org/10.1177/1045389x13507347

21 Sun, Z. X. Theory and application of computer control, Tsinghua University Press, Beijing, (1989).

22 Aulbach, J., Gjonaj, B., Johnson, P. M., Mosk, A. P., and Lagendijk, A. Control of light transmission through opaque scattering media in space and time, Physical Review Letters, 106 (10), 103901, (2011). https://dx.doi.org/10.1103/physrevlett.106.103901

23 Bubnicki, Z. Modern control theory, Springer Science \& Business Media, (2005). https://dx.doi.org/10.1007/3-54028087-1

24 Kirk, D. E. Optimal control theory: an introduction, Courier Dover Publications, (2012). https://dx.doi.org/10.1002/aic.690170452

25 Preumont, A. Vibration control of active structures: an introduction, Springer, (2011). https://dx.doi.org/10.1007/978-94-007-2033-6 